\title{
Caracterização epidemiológica e sociodemográfica da leishmaniose tegumentar americanana na região franco-brasileira
}

\section{Epidemiological and sociodemographic characterization of american tegumentar leishmaniose in the franco-brazilian region}

\footnotetext{
2 Veridiana Barreto do Nascimento

2 Ed Carlos Vasconcelos

3 Jéssica Samara dos Santos Oliveir

4 Sheyla Mara Silva de Oliveira

Universidade Federal do oeste do Pará.

Universidade Federal de Macapá.

Universidade Estadual do Pará.

Universidade de São Paulo.
}

1 Luana Almeida dos Santos luanah.orix@gmail.com

\section{Resumo:}

A leishmaniose tegumentar americana (LTA) é uma doença parasitária, endêmica em 88 países com mais de 12 milhões de pessoas infectadas, caracterizando-se, dessa maneira, como um grave problema de saúde pública. Nesse contexto, esta pesquisa buscou caracterizar o perfil epidemiológico e sociodemográfico de casos notificados e atendidos de leishmaniose tegumentar americana no município do Oiapoque-Amapá, durante os anos de 2013 a 2016. Trata-se de uma pesquisa descritiva, retrospectiva e documental, com abordagem quali-quantitativa. 0 levantamento dos dados foi realizado por meio das fichas de notificação compulsória do Sistema de Informação de Agravos e de Notificação, de janeiro de 2013 a dezembro de 2016 . No período estudado, foram notificados 491 casos de LTA. 0 ano de maior notificação foi 2014 com 40,1\% dos casos, registrando os maiores valores de incidência e prevalência (6,34 e 8,33 casos por 1000 mil habitantes, respectivamente). Após análise dos resultados, foi perceptível que o perfil sociodemográfico da leishmaniose tegumentar americana no munícipio do Oiapoque compreende o sexo masculino, com cor autodeclarada parda, baixa escolaridade, ocupação por atividades de garimpagem e procedentes da zona urbana.

\section{Palavras-chave:}

Enfermagem. Feridas cutâneas. Flebotomíneos. Doença parasitária.

\begin{abstract}
:
American cutaneous leishmaniasis (LTA) is a parasitic disease, endemicin 88 countrieswithmorethan 12 million infected, being thus a serious public health problem. In this context, this research aimed to characterize the clinical, epidemiological and sociodemographic profile of cases reported and attended to American cutaneous leishmaniasis in the municipality of Oiapoque-Amapá, during the years of 2013 to 2016. It is a descriptive, retrospective and documentary research, with a qualiquantitative approach. The data were collected through the compulsory notification sheets of the Health and Medical Information System of 2013, December 2016. $40.1 \%$ of the cases. Observatory in the year 2014 largest number of people and prevalence (6.34 and 8.33 cases per 1000 thousand inhabitants, respectively). After analysis of the results it was noticeable that the sociodemographic profile of the American cutaneous leishmaniasis in the municipality of Oiapoque comprise male, self-declared brown color, low education, working in mining activities and coming from the urban area.
\end{abstract}

\section{Keywords:}

Nursing. Cutaneous wounds. Phlebotomines. Parasitic disease.

\section{Como você deve citar?}

SANTOS, Luana Almeida dos et al.Caracterização epidemiológica e sociodemográfica da leishmaniose tegumentar americanana na região franco-brasileira. Cadernos UniFOA, Volta Redonda, n. 41, p. 107-117, dezembro 2019. 


\section{INTRODUÇÃO}

A Leishmaniose Tegumentar Americana (LTA) se define como uma doença parasitária, endêmica em 88 países, apresentando cerca de 12 milhões de pessoas infectadas, e 350 milhões de pessoas ameaçadas por esse problema, caracterizando-se, assim, como um grave problema de saúde pública em todo o mundo (GRAZIANI; OLIVEIRA; SILVA, 2013; BRASIL, 2017). Destarte, é considerada pela Organização Mundial da Saúde (OMS) como uma das seis mais importantes doenças infecciosas, pelo seu alto coeficiente de detecção e capacidade de produzir deformidades no ser humano (OMS, 2010).

De acordo com Basano e Camargo (2004), no Brasil, existem seis espécies de parasitas causadores da leishmaniose cutânea que estão alocadas em dois subgêneros, Leishmania (L.) e Viannia (V.). São elas: Leishmania (L.) amazonensis, L. (V.) brasiliensis, L. (V.) guyanensis, L. (V.) lainsoni, L. (V.) naiffi e L. (V.) shawi. A LTA atinge o homem ao entrar em contato com focos zoonóticos para o desempenho das atividades de risco (garimpo, derrubadas de matas para construção de estradas, usinas hidrelétricas, extração de madeiras e atividades agropecuárias) que são expostos a áreas de matas com a presença de diversas espécies de flebotomíneos infectados (BRASIL, 2010).

A LTA representa um complexo de doenças com importante entendimento clínico, haja vista que possui uma imensa diversidade epidemiológica. Surgiu por volta de 1855, no Brasil, e era conhecida por "Botão da Bahia" ou "Botão de Biskra". Concomitantemente, a confirmação das formas da leishmaniose (úlceras cutâneas e nasobucofaríngeas) só foi possível em 1909, por Lindenberg, que identificou o parasito no organismo de trabalhadores das áreas de desmatamentos em São Paulo (BASANO E CAMARGO, 2004).

A transmissão da LTA ocorre através da picada dos insetos transmissores infectados. Ressalva-se que não há transmissão de pessoa para pessoa. Por conseguinte, o período de incubação da doença corresponde a dois ou três meses após o contato com os insetos, podendo variar de duas semanas (período curto) a dois anos (período longo) (BRASIL, 2010).

No ciclo de transmissão da LTA, o flebotomíneo constitui a ligação entre os reservatórios e o homem, sendo que este assume um caráter de hospedeiro acidental da LTA. Resumidamente, as leishmânias são consideradas parasitas dimórficos que, ao picarem (fêmeas flebotomíneas) os reservatórios infectados, contraem as formas amastigotas (formas aflageladas), que se modificam para as formas promastigotas (formas flageladas) no intestino dos vetores. Estas, dividem-se no intestino médio e migram para o intestino anterior, onde são regurgitadas pelo mosquito, quando ele iniciar seu novo repasto sanguíneo no hospedeiro. As promastigotas, por sua vez, são as formas infectantes do parasito que interagem com as células do sistema fagocítico mononuclear, perdem o flagelo e, sob a forma amastigota, passam a se multiplicar no interior das células. Assim, a partir da inoculação das formas promastigotas no hospedeiro, tem-se o início de uma complexa relação entre o parasito e a resposta imunológica do hospedeiro infectado. Essa atividade, posteriormente, determinará a forma clínica da LTA (BRASIL, 2007).

O diagnóstico, segundo Brasil (2017), pode ser executado por meios clínicos e laboratoriais. Na forma clínica, têm-se a possiblidade de visualizar as lesões que são geralmente indolores e que costumam se localizar em partes do corpo mais exposta (pernas, braços e face). Apresentam formato arredondado e/ou ovalado, possuem tamanhos que variam de milímetros a centímetros, é eritematosa, infiltrada e com consistência firme. Suas bordas são delimitadas e elevadas com fundo avermelhado.

Já os meios laboratoriais de diagnóstico, incluem a utilização de exames imunológicos e parasitológicos. Nesse contexto, o teste intradérmico (Intradermoreação de Montenegro ou da Leishmania) (IDRM) é o principal exame do grupo imunológico. Nele, tem-se a possiblidade de verificar a resposta de hipersensibilidade celular diferida, que pode ser negativa nas primeiras 4 horas ou após 6 semanas 
do surgimento da lesão cutânea. O IDRM apresenta-se positivo em quase $90 \%$ dos casos e possui vários centímetros de induração e vesiculação no centro da reação, onde podem ocorrer ulceração e necrose local (BRASIL,2017).

Já os exames de ordem parasitológica, incluem a demonstração direta do parasito. São eles: isolamento em cultivo in vitro (meios de cultivo), Isolamento in vivo (inoculações animais) e Reação em cadeia da polimerase (PCR). A demonstração direta do parasito é o procedimento de primeira escolha, haja vista que possui eficácia mais rápida do que os outros, apresenta menor custo e é de fácil aplicação. Para realizar esse procedimento, usa-se a escarificação da borda da lesão e/ou biopsia com fornecimento de fragmento cutâneo. 0 isolamento em cultivo in vitro, por sua vez, é considerado um método que possui a capacidade de identificar o agente etiológico e a espécie do parasito.

Para Brasil (2014), a LTA é uma doença de notificação compulsória, em que todo caso confirmado deve ser notificado e investigado pelos serviços de saúde através da ficha de investigação compulsória padronizada pelo Sistema de Informação de Agravos de Notificação (SINAN). Nesse sentido, o registro é importante para o conhecimento da investigação, bem como para a classificação epidemiológica (caso autóctone ou importado) e acompanhamento dos casos.

Este estudo buscou caracterizar o perfil epidemiológico e sociodemográfico de casos notificados e atendidos de LTA no município do Oiapoque-Amapá, durante os anos de 2013 a 2016.

\section{METODOLOGIA}

Este estudo é definido como um estudo descritivo, retrospectivo, documental, com a abordagem quali-quantitativa. Oiapoque é um dos 16 munícipios que compõem o estado do Amapá, está localizado na mesorregião do norte do Amapá, limitando-se, ao Sul, com os municípios de Calçoene, Serra do Navio e Pedra Branca do Amapari. Ao Norte, delimita-se com a Guiana Francesa. Ao Leste, é banhado pelo Oceano Atlântico e, ao Oeste, faz fronteira com o município de Laranjal do Jari. Apresenta uma área territorial de 22.625, $286 \mathrm{~km}^{2}$, e uma população estimada de 25.514 habitantes (IBGE, 2017). Oiapoque é dividida em 2 distritos: Clevelândia do Norte (área de destacamento militar do exército) e Vila Velha (área de propriedades agroextrativistas) (IBGE, 2017).

A coleta de dados foi realizada através da análise do banco de dados do SINAN e das fichas de notificação (quando disponível na Secretaria Municipal de Saúde), compreendendo o período de janeiro de 2013 a dezembro de 2016.

Para coleta, considerou-se as seguintes variáveis: ano de notificação da doença; sexo; raça/cor; escolaridade (ano do diagnóstico); local de contágio; ocupação/trabalho (durante o diagnóstico); área de residência (durante o diagnóstico).

As informações coletadas das fichas de notificação compulsória dos casos de LTA foram inseridas e organizadas. Assim, utilizou-se estatística descritiva para obtenção das frequências absoluta e relativa. Para quantificar a incidência e a prevalência, usou-se os cálculos estabelecidos por Lima et al. (2003). Quando possível, teste t e o teste de Kruskal-Wallis foram usados para comparação entre médias, seguindo suas respectivas premissas (ZAR, 1999). A pesquisa foi aprovada pelo Comitê de Ética da Universidade de Macapá CAEE: 68584317.2.0000.0003. Número do Parecer: 2.093.053. 


\section{RESULTADOS E DISCUSSÃO}

No período em estudo, foram notificados 491 casos de LTA. A distribuição de casos (Figura 1) mostrou que quase metade dos casos $(40,1 \%)$ foi notificada em 2014; o ano seguinte demonstrou os menores valores, voltando a uma leve trajetória de crescimento entre os anos de 2015 e 2016.

Figura 1 - Distribuição, por ano 191, dos casos leishmaniose tegumentar americana notificados e atendidos no município do Oiapoque, município do Estado de Amapá, no período de 2013 a 2016.

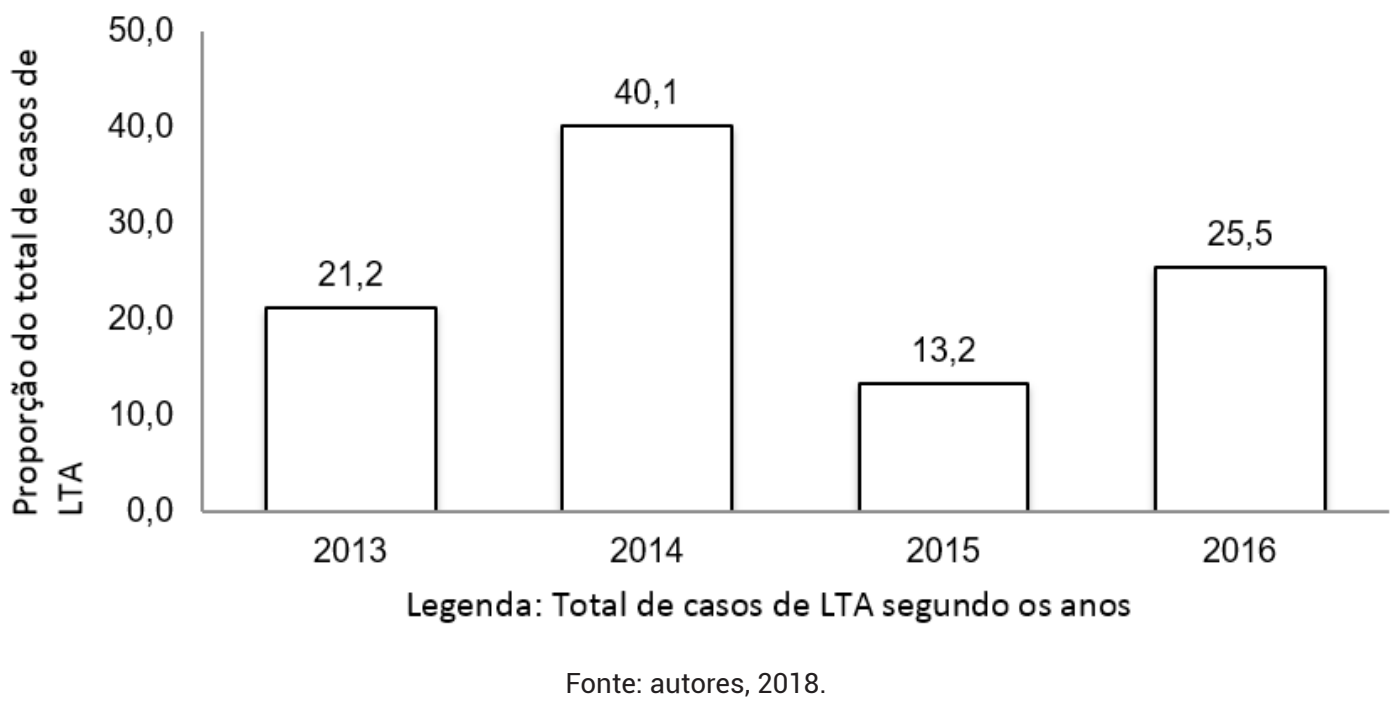

Cruz (2016), ao executar uma pesquisa de caráter epidemiológico no município de Acarape, nos anos de 2012 a 2015, identificou cerca de $38,46 \%$ dos casos de LTA, em 2012; 16,4\%, em 2013; 19,6\%, em 2014; e 25,5\%, em 2015. Salienta-se que esse estudo não corresponde ao período temporal desta pesquisa, porém pode-se tomar como base essa literatura para evidenciar o fato de que a distribuição dos casos de LTA no Oiapoque e em outras localidades propícias ao desenvolvimento da infecção não ocorrem de forma homogênea, considerando a oscilação de casos ao longo dos anos. Portanto, pode-se sugerir que o aumento ou diminuição das notificações de LTA pode ser dependente das características ambientais, regionais e culturais de cada localidade.

Já a Figura 2, apresenta a distribuição dos casos de acordo com o sexo dos pacientes acometidos pela LTA, no período de 2013 a 2016. O sexo masculino foi, significativamente, mais acometido que 0 feminino (em média, quase quatro vezes mais). Considerando os quatro anos juntos, são, em média, 97,5 homens infectados, contra 25,2 mulheres (Teste $t: t=-3,31 ; p=0,01$ ). Em 2014, registrou-se $o$ maior número de casos, tanto para homens quanto para mulheres. 
Figura 2 - Distribuição dos casos 491 de LTA por sexo e ano, atendidos e notificados na cidade do Oiapoque, no período de 2013 a 2016.

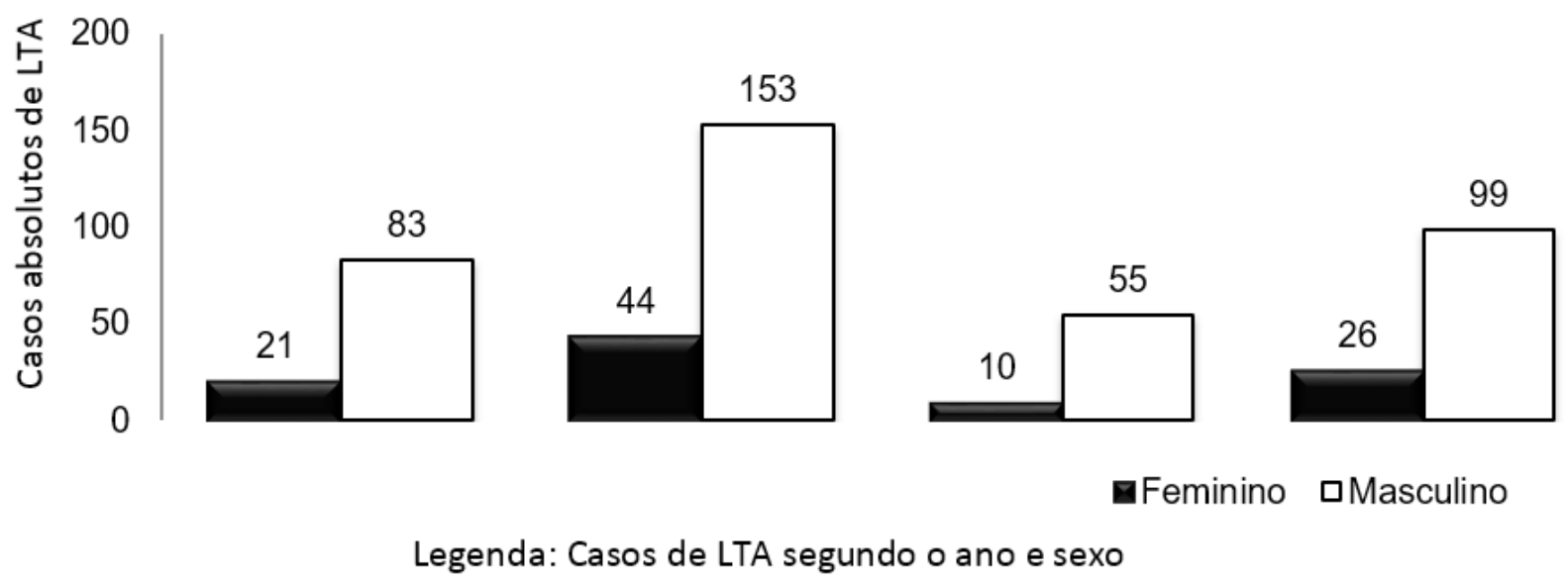

Fonte: autores, 2018.

Para Rodrigues (2008), Guerra et al. (2007) e Oliveira (2011), o sexo masculino apresenta maior predominância para o desenvolvimento da LTA, concordando com os dados deste estudo. Esse fato pode ser explicado, pois os homens apresentam maior exposição ao flebotomíneo infectado, principalmente, quando esses indivíduos estão em atividades laborais que necessitam de sua inserção no habitat natural do vetor. Ao contrário, as mulheres estão menos expostas ao flebotomíneo, pois, na maioria das vezes, desenvolvem trabalhos dentro dos seus próprios lares e, portanto, estariam menos expostas aos vetores transmissores do parasita. 0 fato do município do Oiapoque ser cercado de mata local pode contribuir para que os flebotomíneos atinjam também áreas domiciliares e peridomicilares, onde as mulheres mais se encontram.

A Figura 3 mostra o quantitativo de Incidência e Prevalência anual dos casos de LTA para a área estudada. Assim como os demais perfis, os maiores e menores valores coincidem com os anos de 2014 e 2015 , respectivamente. 
Figura 3 - Taxa de incidência e prevalência anual, dos casos atendidos e notificados de LTA, no munícipio do Oiapoque, durante 2013 a 2016.

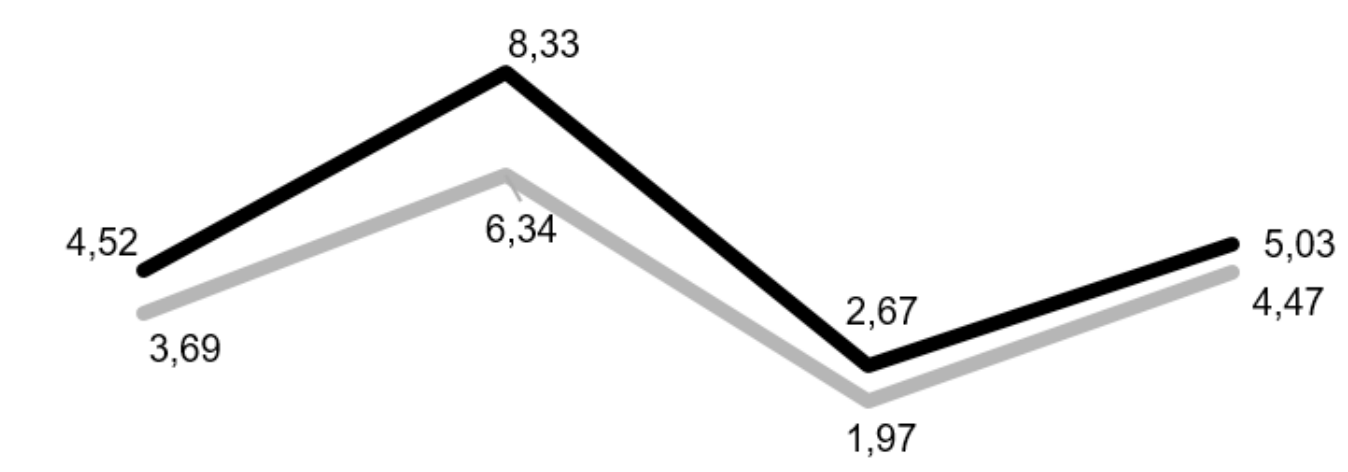

Anos $\quad$ Prevalência Incidência

Legenda:Evolução das taxas de incidência/prevalência segundo o ano

Fonte: autores, 2018.

O Brasil é considerado o terceiro país com o maior número de casos novos em todo o mundo, com uma média 21.000 casos anuais, no período de 2009 a 2013, representando $10 \%$ de todos os casos registrados no mundo, perdendo apenas pela Síria e Afeganistão (OMS, 2010).

A Organização Mundial de Saúde (OMS) estima que cerca de três milhões de pessoas estão acometidas pela leishmaniose tegumentar americana, 12 milhões estão infectados e 350 milhões vivem em áreas de risco. A incidência anual de leishmaniose é de dois milhões de casos e, destes, 1,5 milhão correspondem à leishmaniose tegumentar americana (PONCE E CABALLERO, 2015).

A doença atinge todas as regiões brasileiras e, em 2014, observou-se 20.418 casos novos (BRASIL, 2016). Os valores de incidência encontrados neste estudo são quase cinco vezes maiores, quando comparados com os de Cruz (2010), que registrou índice de 1,04 casos por 1000 habitantes no período da pesquisa.

Os valores de incidência de leishmaniose tegumentar americana encontrados na região expressam um alerta acerca do manejo, prevenção e controle da doença. É de extrema relevância a sensibilização da comunidade, profissionais de saúde no enfrentamento dessa doença que apresenta inúmeras consequências em caso de ocorrência desenfreada, em diferentes contextos.

A OMS afirma que a prevalência da LTA está em torno de 12 milhões no mundo, e que ela apresenta cada vez mais tendências lineares de crescimento, devido, sobretudo, ao desenvolvimento desenfreado das cidades. Sabe-se que, no Brasil, a região norte ainda detém maiores taxas de identificação dessa doença, haja vista que essa região é rodeada de florestas habitat do flebotomíneo (SILVA; MUNIZ, 2009).

Nos dados sociodemográficos, foi observado, na variável raça/ cor dos pesquisados, que a maioria dos pacientes acometidos pela LTA apresentaram a cor parda, com 85,6\%, no ano de 2013; $73,6 \%$, no ano de $2014 ; 47,7 \%$, no ano de $2015 ; 57,6 \%$, no ano de 2016 . Sabe-se que a variável raça/ 
cor está ligada às características regionais de cada localidade, onde houve a notificação da doença, e não a um grupo étnico susceptível, pois a LTA pode acometer tanto em indivíduos brancos, pretos, amarelos, indígenas ou pardos.

Verificou-se resultados parecidos ao desta pesquisa no trabalho realizado na fronteira amazônica, por Oliart-Guzmán et al. (2013), os quais identificaram que 32,6\% da sua amostra (maioria) declararam a raça parda. Em consonância com esses resultados, Batista et al. (2014), ao analisarem os casos de LTA no estado Piauí, entre os anos de 2007 a 2011, notaram que a maioria dos seus pesquisados, também foram classificados com a cor parda.

A escolaridade foi respondida por apenas 169 pacientes. A maioria dos infectados apresentou ensino fundamental $(N=111)$, seguido de ensino médio $(N=53)$ e apenas três tinham ensino superior, e dois eram analfabetos (Tabela 1). No geral, a diferença entre eles foi significativa (Kruskal-Wallis: $\mathrm{H}=$ 11,$75 ; p=0,008$ ). No entanto, comparando a quantidade total de infectados nos quatro anos aos pares (Figura 4), constatou-se que apenas a quantidade de analfabetos e de estudantes do ensino superior e entre quantidade de infectados do ensino fundamental e médio não foram significativamente diferentes.

Tabela 1 - Escolaridade dos portadores de LTA, durante os anos de 2013 a 2016, atendidos no munícipio do Oiapoque.

\begin{tabular}{|c|c|c|c|c|c|c|c|c|}
\hline \multirow{3}{*}{$\begin{array}{c}\text { VARIÁVEIS } \\
\text { ESCOLARIDADE }\end{array}$} & \multicolumn{8}{|c|}{$\operatorname{LTA}(\mathrm{N}=491)$} \\
\hline & \multicolumn{2}{|c|}{2013} & \multicolumn{2}{|c|}{2014} & \multicolumn{2}{|c|}{2015} & \multicolumn{2}{|c|}{2016} \\
\hline & $\mathrm{N}$ & $\%$ & $\mathrm{~N}$ & $\%$ & $\mathrm{~N}$ & $\%$ & $\mathrm{~N}$ & $\%$ \\
\hline Analfabeto (a) & 0 & 0 & 0 & 0 & 1 & 1,5 & 1 & 0,8 \\
\hline Ensino Fundamental & 18 & 17,3 & 18 & 9,1 & 8 & 12,3 & 67 & 53,6 \\
\hline Ensino Médio & 9 & 8,7 & 4 & 2,0 & 6 & 9,2 & 34 & 27,2 \\
\hline Ensino Superior & 0 & 0 & 1 & 0,5 & 0 & 0 & 2 & 1,6 \\
\hline Ignorada & 77 & 74 & 174 & 88,3 & 50 & 76,9 & 21 & 16,8 \\
\hline Total & 104 & 100,0 & 197 & 100,0 & 65 & 100,0 & 125 & 100,0 \\
\hline
\end{tabular}

Fonte: autores, 2018.

Figura 4: Diferenças entre os valores médios de $\mathrm{R}$ aos pares de escolaridade dos portadores de LTA, durante os anos de 2013 a 2016, atendidos no munícipio do Oiapoque, utilizando-se o teste de Kruskal-Wallis. 
Figura 4 - Diferença entre médias de cada escolaridade, dos casos atendidos e notificados de LTA, no munícipio do Oiapoque, durante 2013 a 2016.

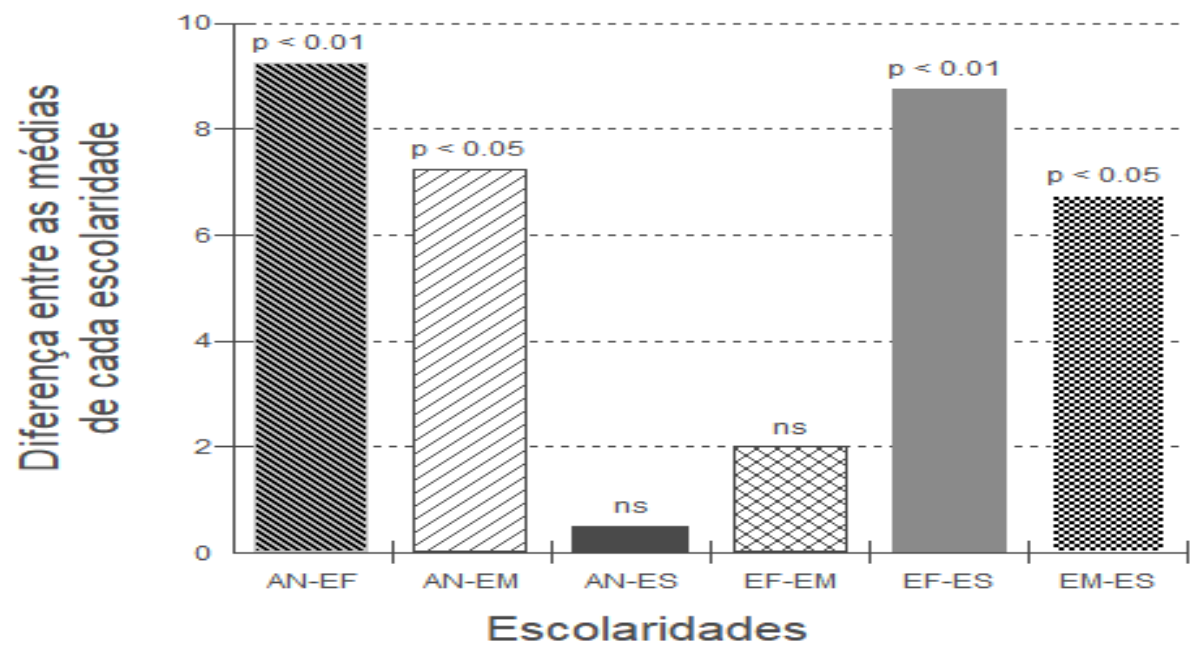

Legenda: $(\mathrm{AN}=$ analfabetos, $\mathrm{EF}=$ ensino fundamental, $\mathrm{EM}=$ ensino médio e $\mathrm{ES}=$ ensino superior $)$ segundo $\mathrm{o}$ ano.

Fonte: autores, 2018.

Maia et al. (2017) também encontraram dados parecidos em um estudo no estado do Acre, os quais concluíram que $64,9 \%$ (maioria) de seu universo amostral caracterizou-se na opção "ensino fundamental".

Quanto à ocupação laboral, a atividade de "Garimpagem" obteve maior ênfase nos casos noticiados, apresentando cerca de $31,7 \%(\mathrm{~N}=33)$, dos casos em 2013,$39 ; 1 \%(\mathrm{~N}=77)$, no ano de $2014 ; 27,7 \%(\mathrm{~N}=18)$, em 2015; e 24,8\% ( $\mathrm{N}=31)$. Chagas et al. (2006) notaram que os indivíduos que tinham atividade laboral próximas ou que mantiveram contato direto com florestas eram os mais vulneráveis à infecção pela LTA. Entretanto, as zonas de moradia dos pacientes com LTA procedentes do munícipio do Oiapoque foi a zona urbana, expondo cerca de 89,4\% dos casos, em 2013; 79,7\% dos casos, em 2014; 69,2\%, em 2015; e 79,2\%, no ano de 2016. Resultados parecidos foram observados por Xavier et al. (2016) e Graziani et al. (2013). Nestes, a maioria ( $79 \%$ e $68 \%$, respectivamente) dos acometidos pela parasitose em questão era oriundo da zona urbana.

Em relação à zona de moradia, verificou-se resultados parecidos no trabalho de Xavier, Mendes Rossi-Barbosa (2016), executado na cidade de Montes Claros, MG. No período de 2010 a 2013, notou-se que a maioria (79\%) dos seus pesquisados eram oriundos da zona urbana. Outro resultado análogo ao desse estudo foi identificado por Graziani, Oliveira e Silva (2013), onde encontrou-se que $68 \%$ da sua amostra analisada era procedente da zona urbana.

Segundo Basano e Camargo (2004), existem dois padrões epidemiológicos para retratar a transmissão da LTA. Um desses padrões está associado à crescente ocupação de territórios com matas fechadas com a presença de reservatórios do protozoário em animas silvestres. 0 segundo padrão resulta das transformações que o processo de urbanização trouxe às cidades, ou seja, a urbanização propiciou condições favoráveis ao cultivo dos focos da endemia, fortalecendo a persistência e o desenvolvimento da LTA, além do processo de domicialização dos flebotomíneos, resultando no crescente aumento da vulnerabilidade para os indivíduos que habitam um desses dois tipos de locais propícios à transmissão. 


\section{CONCLUSÃo}

O presente estudo se propôs a identificar o perfil epidemiológico e sociodemográfico dos casos de Leishmaniose Tegumentar Americana no município de Oiapoque-Amapá, entre os anos de 2013 a 2016, através da análise de 491 fichas de notificação compulsória. Assim, foi possível contribuir com o perfil da LTA no cenário nacional.

Após análise dos resultados, foi perceptível que o perfil sociodemográfico da LTA no munícipio do Oiapoque é composto pelo sexo masculino, com cor autodeclarada parda, baixa escolaridade, ocupação por atividades de garimpagem e procedentes da zona urbana. Foram identificados a incidência e a prevalência dos casos de LTA oriundos Amazônia franco-brasileira, o que auxilia na tomada de decisões e o enfrentamento dessa problemática.

Salienta-se a necessidade do desenvolvimento de Educação em Saúde para os grupos de riscos quanto à prevenção e à transmissão da doença, a fim de minimizar o desenvolvimento e propagação das notificações no munícipio do Oiapoque e das cidades circunvizinhas.

Mediante os dados aqui apresentados, este trabalho torna-se também importante para a área da Enfermagem, haja vista que são os profissionais que estão diretamente ligados à comunidade nos três níveis de atenção à saúde devendo, sobretudo, conhecer os aspectos epidemiológicos das patologias, pois é sobre esse fator que são tomadas as futuras políticas de saúde, bem como as medidas de prevenção que serão executadas pela própria Enfermagem. 


\section{REFERÊNCIAS}

AZEVEDO, A. C. R.; SOUZA, N. A.; MENESES, C. R. V.; COSTA,W. A.; COSTA, S. M.; LIMA, J. B.; RANGEL, E. F. Ecology of Sand Flies (Diptera: Psychodidae: Phlebotominae) in the North of the State of Mato Grosso, Brazil. Memórias do Instituto Oswaldo Cruz, 2002. 97: 459-464.

CHAGAS, A. C; PESSOA, F. A. C; MEDEIROS, J. F; PYDANIEL. V; MESQUITA, E. C; BALESTRASSI, D.A. Leishmaniose Tegumentar Americana (LTA) em uma vila de exploração de minérios-Pitinga, município de Presidente Figueiredo. Amazonas, Brasil. Revista brasileira Epidemiologia[online]. vol. 9, n. 2, pp. 186-192. ISSN 1415-790X. 2006, http://dx. Doi. Org/10.1590/S1415-790X2006000200005.

BASANO, S. A.; CAMARGO, L. M. A. Leishmaniose tegumentar americana: histórico, epidemiologia e perspectivas de controle. Revista Brasileira Epidemiologia. Rondônia, 2004.

BRASIL, Ministério da Saúde. Sistema de Informação de Agravos de Notificação. 2016 Disponível em: http://www2.datasus.gov.br/DATASUS/index.php?area=0203\&id=29878153. Acesso em: 27 mar. 2017.

BRASIL. Ministério da Saúde. Secretaria de Vigilância em Saúde. Manual de vigilância da leishmaniose tegumentar americana [Internet]. 2007. Disponível em: http://www.saude.gov.br. Acesso em: 27 mar. 2017.

BRASIL. Ministério da Saúde. Secretaria de Vigilância em Saúde. Manual de Vigilância da Leishmaniose Tegumentar. Série de Manuais A. 2. ed. atual. Brasília, 2010.

BRASIL. Ministério da Saúde. Secretaria de Vigilância em Saúde. Guia de Vigilância em Saúde. Série de Manuais II. Brasília, 2014.

BRASIL. Ministério da Saúde. Secretaria de Vigilância em Saúde. Departamento de Vigilância das Doenças Transmissíveis. Manual de vigilância da leishmaniose tegumentar [recurso eletrônico]. $n^{\circ} 1$. Brasília, 2017.

BATISTA, F. M. A.; MACHADO, F. F. O. A; SILVA, J. M. O; MITTMAN. J; BARJA. P. R; SIMIONI, A. R; Leishmaniose: perfil epidemiológico dos casos notificados no estado do Piauí entre 2007 e 2011. Revista Univap. v. 20, n. 35. 2014.

CRUZ, C. F. R. Leishmaniose Tegumentar Americana (LTA) no município de Bandeirantes - Paraná, entre 2000 e 2009. Dissertação (Mestre em Saúde Pública) - Faculdade de Saúde Pública, USP, São Paulo, 2010.

CRUZ, G. S. Leishmaniose tegumentar americana: aspectos clínicos, epidemiológicos e influência de fatores predisponentes. 2016. Monografia (Graduação de Enfermagem) - Universidade da Integração Internacional da Lusofonia Afro Brasileira. Acarape.

GRAZIANI, D.; OLIVEIRA,V. A. C.; SILVA, R. C. Estudo das características epidemiológicas da leishmaniose tegumentar americana no estado de goiás, brasil, 2007-2009. Revista Patologia Tropical. v. 42, n. 4, p. $417-424,2013$.

GUERRA, J. A. O.; RIBEIRO, J. A. S.; COELHO, L. I. A. R. C.; BARBOSA, M. G. V.; PAES, M. G. Epidemiologia da Leishmaniose Tegumentar na comunidade São João, Manaus, Amazonas, Brasil. Cadernos de Saúde Pública, 22: 2319-2327. 
GUERRA, J. A. O. et al. Leishmaniose tegumentar americana em crianças: aspectos epidemiológicos de casos atendidos em Manaus, Amazonas, Brasil. Cadernos de Saúde Pública, Rio de Janeiro, v. 23, n. 9, p. 2215-2223, 2007.

INSTITUTO BRASILEIRO DE GEOGRAFIA E ESTATÍSTICA (IBGE). Informações estatísticas [Internet]. Estado do Amapá. Disponível em: http://cidades.ibge.gov.br/brasil/ap/panorama/2017. Acesso em: 22 nov. 2017.

LIMA J. R. C.; PORDEUS, A. M. J.; ROUQUAYROL, M. Z. Medida de Saúde Coletiva. In: Rouquayrol MZ. Epidemiologia e Saúde. Rio de Janeiro: MEDSI; 2003.p.25-64.

MAIA, J. A. et al. Características sociodemográficas de pacientes com leishmaniose tegumentar americana. Revista Enfermagem Contemporânea. v. 6, n. 2, p. 25-32, 2017.

OLIART-GUZMÁN, H. et al. Características epidemiológicas da leishmaniose tegumentar americana na fronteira amazônica: estudo retrospectivo em Assis Brasil, Acre. Rev Patol Trop. v. 42, n. 2, p.187-200. 2013.

OLIVEIRA, A. C. M. Caracterização epidemiológica da Leishmaniose Tegumentar Americana no município de Rio Branco-Acre no período de 2000 a 2008. 2011. Dissertação (Mestrado em Saúde Pública) - Escola Nacional de Saúde Pública, Rio de Janeiro.

OMS. Lucha contra las leishmaniasis. Série de Informes Técnicos 949. v.793. Ginebra, 2010.

PONCE BAHARONA, O.A; CABALlero CAStRo H.R. Leishmaniasis cutánea. Act Ped Hond. 2015; 5(1):365-369

RODRIGUES, A. C. E. Características Epidemiológicas e Distribuição Espacial da Enzootia Canina de Leishmaniose Visceral na Cidade de Teresina - Piauí, no período de 2003 - 2006. 2008. Dissertação (Mestrado em Saúde Pública) - Fundação Oswaldo Cruz, Teresina.

SILVA, N. S.; VIANA, A. B.; CORDEIRO, J. A; CAVASINI, C. E.; 1999. Leishmaniose tegumentar americana no Estado do Acre. Revista de Saúde Pública, 33: 554-559.

SILVA, N. S.; MUNIZ, V. D. Epidemiologia da leishmaniose tegumentar americana no estado do Acre, Amazônia brasileira. Caderno de saúde pública. Rio de Janeiro. v. 25, n.6, p. 1325-1336. 2009.

XAVIER, K. D.; MENDES, F. C. F.; ROSSI-BARBOSA, L. A. R. Leishmaniose tegumentar americana: estudo clínico-epidemiológico. Revista da Universidade Vale do Rio Verde, Três Corações. v. 14, n. 2, p. 1210 1222. 2016.

ZAR, J. H. Biostatistical Analysis. 4. ed. New Jersey: Prentice-Hall, 1999. 